\title{
Generic phase diagram of spin relaxation in solids and the Loschmidt echo
}

\author{
Gábor Csősz $\odot,{ }^{1, *}$ Lénárd Szolnoki, ${ }^{1, *}$ Annamária Kiss, ${ }^{2,1}$ Balázs Dóra, ${ }^{3}$ and Ferenc Simon $\oplus^{1,4, \dagger}$ \\ ${ }^{1}$ Department of Physics, Budapest University of Technology and Economics and MTA-BME Lendület Spintronics Research Group \\ (PROSPIN), P.O. Box 91, H-1521 Budapest, Hungary \\ ${ }^{2}$ Institute for Solid State Physics and Optics, Wigner Research Centre for Physics, P.O. Box 49, H-1525 Budapest, Hungary \\ ${ }^{3}$ Department of Theoretical Physics and MTA-BME Lendület Topology and Correlation Research Group, Budapest University of Technology \\ and Economics, 1521 Budapest, Hungary \\ ${ }^{4}$ Laboratory of Physics of Complex Matter, École Polytechnique Fédérale de Lausanne, Lausanne CH-1015, Switzerland
}

(Received 4 November 2019; revised 26 February 2020; accepted 14 June 2020; published 13 July 2020)

\begin{abstract}
The spin relaxation time in solids is determined by several competing energy scales and processes, and distinct methods are called for to analyze the various regimes. We present a stochastic model for the spin dynamics in solids which is equivalent to solving the spin Boltzmann equation and takes the relevant processes into account on an equal footing. The calculations reveal yet unknown parts of the spin-relaxation phase diagram, where strong reversible spin dephasing occurs in addition to spin relaxation. Spin-relaxation times are obtained for this regime by introducing the numerical Loschmidt echo. This allows us to construct a generic approximate formula for the spin-relaxation time, $\tau_{\mathrm{s}}$, for the entire phase diagram, involving the quasiparticle scattering rate, $\Gamma$, spin-orbit coupling strength, $L$, and a magnetic term, $\Delta_{Z}$ due to the Zeeman effect. The generic expression reads as $\hbar / \tau_{\mathrm{s}} \approx \Gamma L^{2} /\left(\Gamma^{2}+L^{2}+\Delta_{\mathrm{Z}}^{2}\right)$.

DOI: 10.1103/PhysRevResearch.2.033058
\end{abstract}

\section{INTRODUCTION}

The emerging field of spintronics [1,2] envisions to employ the electron spin as an information carrier instead of the usual charge degree of freedom, thus allowing for more efficient and high-performance future informatics devices. This potential leads to renewed efforts for both the theoretical understanding of spintronics relevant phenomena [2,3] and also the exploration of novel materials for this purpose. In particular, two-dimensional materials, such as graphene [4], appear to be excellent spintronics candidates [5-7].

Whether a material can be successfully employed in spintronics is decided by the magnitude of the so-called spinrelaxation time, $\tau_{\mathrm{s}}$, and the related spin-diffusion length, $\delta_{\mathrm{s}}$. These are the analogous quantities to the charge-carrier lifetime and carrier diffusion length in semiconductors. There is an overall consensus that spin relaxation is dominated by spin-orbit coupling (SOC) effects [2,3]. The Elliott-Yafet $[8,9]$ and Dyakonov-Perel (DP) $[10,11]$ theories explain spin relaxation in metals and semiconductors with and without inversion symmetry (e.g., GaAs), respectively. Although these two descriptions are formulated differently, these were recently brought to a common mathematical basis [12].

\footnotetext{
${ }^{*}$ These authors contributed equally to this work.

†.simon@eik.bme.hu

Published by the American Physical Society under the terms of the Creative Commons Attribution 4.0 International license. Further distribution of this work must maintain attribution to the author(s) and the published article's title, journal citation, and DOI.
}

Modern advances in the description of spin relaxation aim at accurately determining $\tau_{\mathrm{s}}$ with first-principles methods including the details of the crystal, band structure, and electronphonon coupling [13-17]. However, it turned out recently that conventional spin-relaxation theories require refinement $[12,18,19]$, which also affects the first-principles descriptions. A representative example is the case of graphite, where firstprinciples prediction gives a temperature dependence of $\tau_{\mathrm{s}}$ that is opposite to the experimentally observed one [15].

Spin-relaxation time (and the corresponding spinrelaxation rate, $\left.\Gamma_{\mathrm{s}}=\hbar / \tau_{\mathrm{s}}\right)$ is strongly influenced by the momentum scattering time, $\tau$ (or the strength of quasiparticle scattering, $\Gamma=\hbar / \tau)$, by the magnitude of an external magnetic field and by the spin-orbit coupling. The magnetic field induced Zeeman splitting is characterized by $\Delta_{\mathrm{Z}}$ and the SOC strength is characterized by its Fermi surface averaged effective matrix element, $L$, but it can be associated with a built-in, SOC-related magnetic field. The different spin-relaxation regimes are summarized in Fig. 1 along with the corresponding band structure.

The DP regime is highlighted in the figure, which occurs when $\Gamma \gg\left(L, \Delta_{\mathrm{Z}}\right)$. This is the historically best known $[10,11]$ and experimentally most intensively studied regime in zinc-blende-type semiconductors [20,21]. The large magnetic field limit $\Delta_{\mathrm{Z}} \gg(\Gamma, L)$ was studied experimentally in Ref. [20] and described theoretically in Refs. [18,22,23]. Finally, the large $L$ regime was qualitatively described in Ref. [2] to give rise to a strongly nonexponential spin decay or dephasing. This latter regime was experimentally observed in high-mobility GaAs [24,25].

The decay of spin coherence can be due to irreversible and reversible processes. The former involves a memory loss and an entropy increase, whereas the latter does not involve a full 


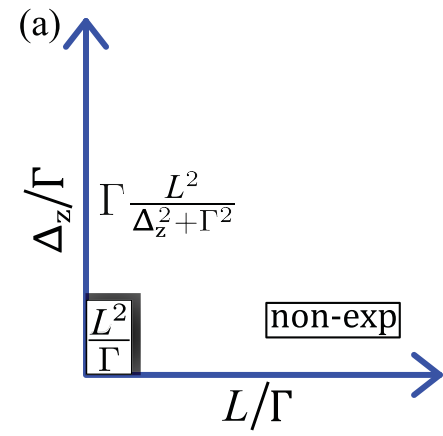

(b)

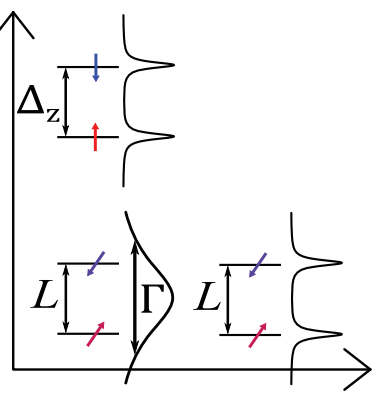

FIG. 1. (a) Phase diagram of spin relaxation for materials without inversion symmetry. The DP [10,11] regime is indicated by a shaded box in the bottom left corner. The other known result for the $\Gamma-\Delta_{Z}$ line (Ref. [18]) is also given. The large $L$ regime leads to a nonexponentially dephasing regime $[2,19]$, which is tackled herein. (b) Energy level schemes for the different relaxation regimes; the gaps, spin degeneracy, and the quasiparticle spectral function are indicated. Vertical and tilted arrows depict spin and SOC eigenstates, respectively.

memory loss. The concept of the Loschmidt echo [26] was introduced for such situations in a famous Gedankenexperiment to allow separation of the two effects. In practice, the most successful realization of the Loschmidt echo is the spin echo [27].

The presence of reversible dephasing smears the irreversible effects and the determination of the spin-relaxation time. This motivated us to develop a numerical approach to the dynamics of spins of noninteracting electrons, which includes momentum scattering and spin precession under the action of an external and the SOC-related magnetic fields. The method provides the quantum trajectories [28-30] for individual spins and is shown to be equivalent to the exact solution of the spin Boltzmann equation. Spin-relaxation time can be obtained even in the presence of strong reversible dephasing with the introduction of a numerical Loschmidt echo. This allowed us to construct the full phase diagram of $\left(\Gamma, L, \Delta_{\mathrm{Z}}\right)$ and we find that a generic formula

$$
\Gamma_{\mathrm{s}} \approx \Gamma \frac{L^{2}}{\Gamma^{2}+L^{2}+\Delta_{\mathrm{Z}}^{2}}
$$

fits well the data for the entire phase diagram.

\section{RESULTS AND DISCUSSION}

The stochastic model

We consider the time evolution of electrons in the presence of spin-orbit coupling and scattering on impurities, the two basic ingredients for spin relaxation. The total Hamiltonian reads as

$$
H=H_{0}+H_{\text {imp }}+H_{\text {SOC }}+H_{\mathrm{Z}} \text {. }
$$

Here, the kinetic energy $\left(H_{0}\right)$ and impurity potential $\left(H_{\mathrm{imp}}\right)$ leave the spin intact, and the latter is responsible for the finite lifetime of electrons. The spin-orbit coupling term, $H_{\text {SOC }}=\sum_{k} \mathbf{b}(\mathbf{k}) \cdot \boldsymbol{\sigma}$, and the Zeeman term, $H_{\mathrm{Z}}$, due to an external magnetic field, involve the electron spin, and the explicit structure of $\mathbf{b}(\mathbf{k})$ is determined by the actual spin-orbit
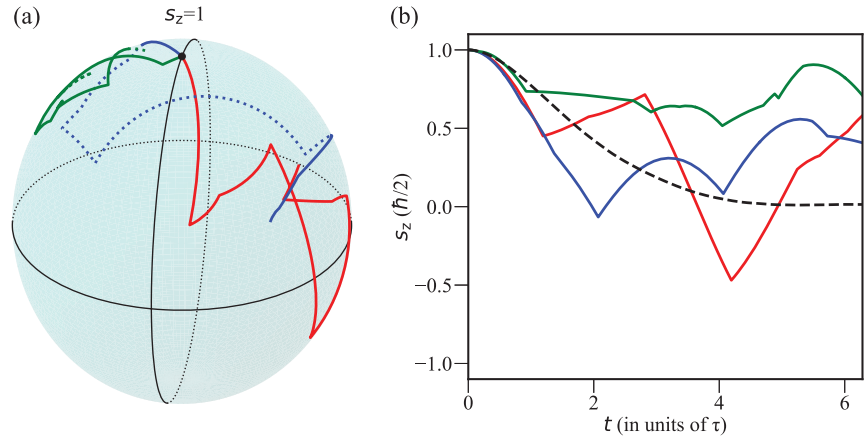

FIG. 2. (a) Quantum trajectories of three individual spins on the Bloch sphere (dotted curves are trajectories on its back). All spins are polarized at $t=0$ to $s_{z}=1$, and they evolve under the action of the external and SOC magnetic field. Elapsed time between two momentum scatterings follows a Poisson distribution with an expectation value of $\tau$. (b) Time evolution of the $s$ component for individual spins and the dashed curve is the ensemble-averaged time evolution of $s_{z}$.

coupling process. Fabian et al. (Ref. [31]) showed that a proper description of spin dynamics can be obtained by treating the electron spin quantum mechanically and the electron momentum quasiclassically. The Liouville-von Neumann equation leads, for the above Hamiltonian, to the socalled spin Boltzmann equation [31]:

$$
\frac{\partial \rho_{\boldsymbol{k}}}{\partial t}-\frac{1}{i \hbar}\left[H_{\mathrm{SOC}}+H_{\mathrm{Z}}, \rho_{\boldsymbol{k}}\right]=\sum_{\boldsymbol{k}^{\prime}} W_{\boldsymbol{k} \boldsymbol{k}^{\prime}}\left(\rho_{\boldsymbol{k}^{\prime}}-\rho_{\boldsymbol{k}}\right),
$$

where $\rho_{\boldsymbol{k}}$ is the $2 \times 2$ spin density matrix, which describes the spin of an electron with wave number $\boldsymbol{k}$. The second term on the left side gives spin evolution under the action of the spin-orbit coupling and Zeeman terms. $W_{\boldsymbol{k} \boldsymbol{k}^{\prime}}$ is the $k \rightarrow k^{\prime}$ scattering probability per unit time due to impurity potential which obeys the detailed balance. The spin expectation value, $\boldsymbol{s}_{\boldsymbol{k}}=\operatorname{Tr}\left[\rho_{\boldsymbol{k}} \boldsymbol{\sigma}\right]$, satisfies

$$
\frac{\partial \boldsymbol{s}_{\boldsymbol{k}}}{\partial t}=\boldsymbol{\Omega}(\boldsymbol{k}) \times \boldsymbol{s}_{\boldsymbol{k}}+\sum_{\boldsymbol{k}^{\prime}} W_{\boldsymbol{k} \boldsymbol{k}^{\prime}}\left(\boldsymbol{s}_{\boldsymbol{k}^{\prime}}-\boldsymbol{s}_{\boldsymbol{k}}\right) .
$$

Here, we introduced the $\boldsymbol{\Omega}(\boldsymbol{k})$ Larmor (angular) frequency, given by $H_{\mathrm{SOC}}+H_{\mathrm{Z}}=\frac{\hbar}{2} \boldsymbol{\Omega}(\boldsymbol{k}) \boldsymbol{\sigma}$, which reflects that the electron spins precess under the action of the external and SOCrelated, built-in magnetic fields. In principle, the numerical solution of Eq. (4) could provide the full spin dynamics for various values of the momentum scattering rate, external magnetic field, and SOC strength.

Our stochastic or Monte Carlo (MC) model considers a spin ensemble with different k's where all spins are initially polarized along the $z$ axis on the Bloch sphere. The spins evolve independently and they undergo Larmor precession between two momentum scattering events. Momentum scattering randomizes $\mathbf{k}$ and precession continues with a new Larmor precession vector. The elapsed time between two momentum scatterings follows a Poisson distribution, with an expectation value of $\tau_{\boldsymbol{k}}=\left(\sum_{\boldsymbol{k}^{\prime}} W_{\boldsymbol{k} \boldsymbol{k}^{\prime}}\right)^{-1}$. Figure 2 shows the quantum trajectories [28-30] of three individual electron spins on the Bloch sphere along with the ensemble-average value of $s_{z}$. 


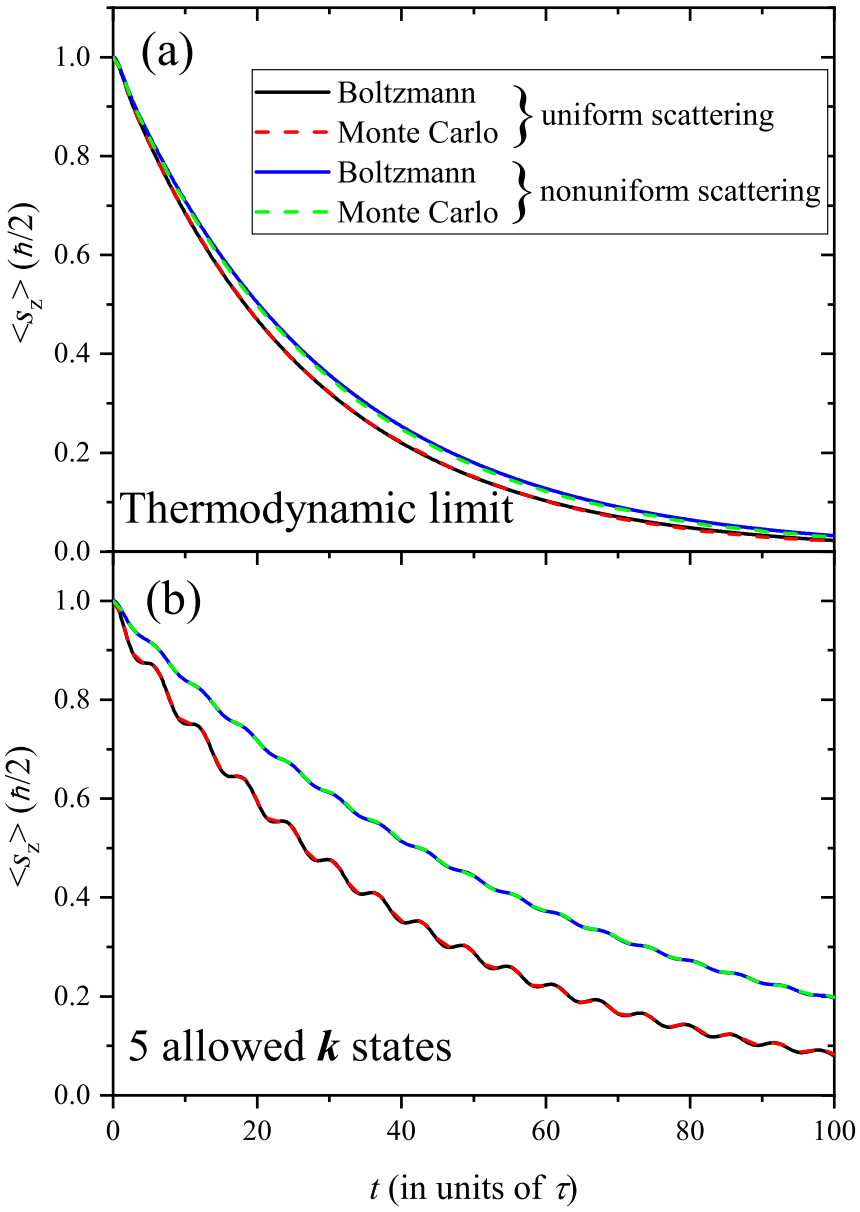

FIG. 3. (a) Comparison of the numerical solution of the spin Boltzmann equation and the Monte Carlo-based method for more than a thousand allowed $\boldsymbol{k}$ states, which is equivalent to the thermodynamic limit. $W_{\boldsymbol{k} \boldsymbol{k}^{\prime}}$ is constant for all $\boldsymbol{k}, \boldsymbol{k}^{\prime}$ for the uniform scattering and it is weighted with $\cos \theta$ ( $\theta$ being the angle of $\boldsymbol{k}$ and the $z$ direction) in the nonuniform case. (b) The same comparison but the number of allowed $\boldsymbol{k}$ states is five, which are given in the text. For all calculations $\Delta=1, \Gamma=1$, and $L=1$ was used.

The time evolution of an individual spin in state $\boldsymbol{k}$ during a short $\Delta t$ time interval is

$$
\boldsymbol{s}_{\boldsymbol{k}}(t+\Delta t)=\left(1-\frac{\Delta t}{\tau_{\boldsymbol{k}}}\right) \boldsymbol{U}_{\boldsymbol{k}}(\Delta t) \boldsymbol{s}_{\boldsymbol{k}}(t)+\Delta t \sum_{\boldsymbol{k}^{\prime}} W_{\boldsymbol{k}^{\prime} \boldsymbol{k}} \boldsymbol{s}_{\boldsymbol{k}^{\prime}}(t),
$$

where $\boldsymbol{U}_{\boldsymbol{k}}(\Delta t) \boldsymbol{s}_{\boldsymbol{k}}(t)=\boldsymbol{s}_{\boldsymbol{k}}(t)+\Delta t \boldsymbol{\Omega}(\boldsymbol{k}) \times \boldsymbol{s}_{\boldsymbol{k}}(t)$. The first term on the right-hand side describes when the spin, $s_{k}$, does not scatter out from $\boldsymbol{k}$ during $\Delta t$. The second corresponds to scattering-in from $\boldsymbol{k}^{\prime}$ states. Assuming detailed balance $\left(W_{k^{\prime} k}=W_{k k^{\prime}}\right)$ and retaining terms in the first order of $\Delta t$ yields directly the spin Boltzmann equation of Eq. (4) in the infinitesimal limit of the time step. We note that this consideration essentially mimics the derivation of the Lindblad equation in Ref. [32], and the dynamics of a spin coupled to a Markovian bath are also treated in standard textbooks such as, e.g., Ref. [33].

In Fig. 3, we compare the result of the Monte Carlo calculations with the solution of the spin Boltzmann equation.
Figure 3(a) gives two examples: one with a uniform scattering (i.e., $W_{k k^{\prime}}$ constant for all $\boldsymbol{k}, \boldsymbol{k}^{\prime}$ ) and one with a nonuniform scattering. For the latter, $W_{k k^{\prime}}$ is weighted by $\cos \theta$, where $\theta$ is the angle between $\boldsymbol{k}$ and the $z$ direction. The calculations were performed for more than 1000 allowed $\boldsymbol{k}$ states which were spread uniformly over the Fermi surface.

Figure 3(b) shows the result of both types of calculations for five allowed $\boldsymbol{k}$ states only: the "North Pole" (NP), "South Pole" (SP), and three points along the "Equator" (E1, E2, and E3) which form an equilateral triangle on the Fermi surface. Again, $W_{\boldsymbol{k} \boldsymbol{k}^{\prime}}$ is constant for the uniform scattering and $W_{\mathrm{NP} \rightarrow \mathrm{SP}}: W_{\mathrm{NP} / \mathrm{SP} \rightarrow \mathrm{E}}=3: 1$ for the nonuniform scattering model.

Remarkably, we find no difference between solving the spin Boltzmann equation and the result of the Monte Carlo model for either case. Therefore the Monte Carlo model gives an accurate description of the spin dynamics, irrespective of the $\boldsymbol{k}$ distribution and the uniformity of the scattering.

Besides being equivalent, the MC method is numerically more effective as the Larmor precession between scattering events can be calculated analytically without resorting to numerics. In contrast, solving the spin Boltzmann equation for a large magnetic field (external or SOC related) requires the use of a small time discretization.

The most important advantage of the MC method is that it tracks the individual quantum trajectories of electron spins, whereas the spin Boltzmann equation inherently provides the ensemble-average values only, similarly to the Schrödinger equation. The MC method is particularly advantageous when we are interested in single events and quantum leaps happening in individual quantum systems, e.g., in mesoscopic systems where statistical fluctuations are important in understanding and analyzing individual measurements [30]. We employ the MC method in the following to study yet unexplored parts of the spin-relaxation phase diagram as a function of $\left(\Gamma, L, \Delta_{\mathrm{Z}}\right)$.

\section{THE RELATION BETWEEN THE SPIN BOLTZMANN EQUATION AND THE BLOCH EQUATIONS}

The phenomenological Bloch equations describe the time evolution of a spin ensemble under the action of an external DC magnetic field and an additional AC magnetic field. The latter is used in magnetic resonance experiments and is usually polarized perpendicularly to the DC field. The Bloch equations are written for the magnetization $\boldsymbol{M}$, which is the ensemble-averaged magnitude of the spin magnetic dipole moments per unit volume. These read

$$
\begin{aligned}
& \frac{d M_{x}(t)}{d t}=\gamma[\boldsymbol{M}(t) \times \boldsymbol{B}(t)]_{x}-\frac{M_{x}(t)}{T_{2}}, \\
& \frac{d M_{y}(t)}{d t}=\gamma[\boldsymbol{M}(t) \times \boldsymbol{B}(t)]_{y}-\frac{M_{y}(t)}{T_{2}}, \\
& \frac{d M_{z}(t)}{d t}=\gamma[\boldsymbol{M}(t) \times \boldsymbol{B}(t)]_{z}-\frac{M_{z}(t)-M_{0}}{T_{1}} .
\end{aligned}
$$

Here, the Larmor vector due to the external magnetic fields (either DC, AC or both) is identified as $\boldsymbol{\Omega}(\boldsymbol{t})=\gamma \boldsymbol{B}(t)$, where $\gamma$ is the gyromagnetic ratio of electrons. The $T_{1}$ and $T_{2}$ are the so-called longitudinal and transversal relaxation times. $M_{0}$ 
appears when the DC magnetic field is along the $z$ axis. The phenomenological relaxation times describe that following an excitation the respective magnetizations return to their equilibrium values, which is $M_{0}$ for the $M_{z}$ component and 0 for $M_{x}$ and $M_{y}$. In a zero external magnetic field, the $T_{1}$ and $T_{2}$ distinction vanishes.

We recognize a clear analogy between the spin Boltzmann equation and the Bloch equations. Although the spin Boltzmann equation contains the external magnetic field and the $\mathbf{k}-$ dependent built-in SOC-related magnetic fields altogether, the latter give rise to the phenomenological relaxation times $\left(T_{1}\right.$ and $T_{2}$ ) in combination with the momentum relaxation events (which are described by the $W_{\mathbf{k}^{\prime} \mathbf{k}}$ terms in the spin Boltzmann equation. The external magnetic field appears unchanged in the Bloch equations.

Strictly speaking, this is only valid for the case when the spin magnetization decays exponentially according to the spin Boltzmann equation. However, the effect of reversible dephasing can be also included in the Bloch equations by introducing, e.g., spatial dependence of the local DC magnetic fields (e.g., due to the inhomogeneity of the magnet) or particle orientation dependent $\gamma$ (or $g$ factor) in a powder sample. The spin Boltzmann equation can also be amended with the diffusion term, whose analog is known as the Bloch-Torrey equations.

\section{Nonexponentional spin relaxation}

We apply the MC method for the Dresselhaus SOC Hamiltonian in three dimensions, where the Larmor (angular) frequency vectors read in $k$ space $\left[\mathbf{k}=\left(k_{x}, k_{y}, k_{z}\right)\right]$

$$
\mathbf{b}(\mathbf{k})=\frac{L^{\prime}}{2 k_{\mathrm{F}}^{3}}\left[k_{x}\left(k_{y}^{2}-k_{z}^{2}\right), k_{y}\left(k_{z}^{2}-k_{x}^{2}\right), k_{z}\left(k_{x}^{2}-k_{y}^{2}\right)\right],
$$

where $L^{\prime}$ is the strength of the SOC in energy units and $k_{\mathrm{F}}$ is the Fermi wave number. The Fermi surface averaging of $L^{\prime}$ gives $L^{2}=L^{\prime 2} \frac{4}{35}$.

We first consider a zero magnetic field, i.e., $\Delta_{\mathrm{Z}}=0$. The condition of the DP description $[10,11]$ is $\Gamma \gg L$, when the MC method gives spin relaxation with a single exponent as $\Gamma_{\mathrm{s}}=\frac{L^{2}}{\Gamma}$. However, the situation drastically changes when $L$ is the leading term and a nonexponential spin decay is observed. This regime was first described in Ref. [2]: it was pointed out that a significant reversible dephasing, rather than relaxation, takes place on a timescale of $1 / \Delta \Omega$, where the latter is the spread in the Larmor frequency distribution. The qualitative reason for reversible dephasing is that the spins precess by a large angle, $\bar{\Omega} \tau \gg 1$, before a momentum scattering takes place ( $\bar{\Omega}$ is the mean value of the Larmor frequency). The reversible dephasing is in fact a procedure without memory loss, which is followed by a truly irreversible spin decay after several $\tau$ elapses $[34,35]$.

The corresponding spin decay is shown in Fig. 4(a) (solid black line). The simulation was performed for an ensemble of $N=10^{5}$ electrons and a nominal value of the Larmor precession frequency of $\hbar \Omega / \Gamma=L^{\prime} / \Gamma=100$. Clearly, the data shows a rapid reversible dephasing, which is followed by an exponential-like tail for longer times. Its Fourier transform, $\mathcal{S}(\omega)$, is presented in Fig. 4(b): it shows a peak function at $\omega=0$ and two side-lobe structures, which are due to the
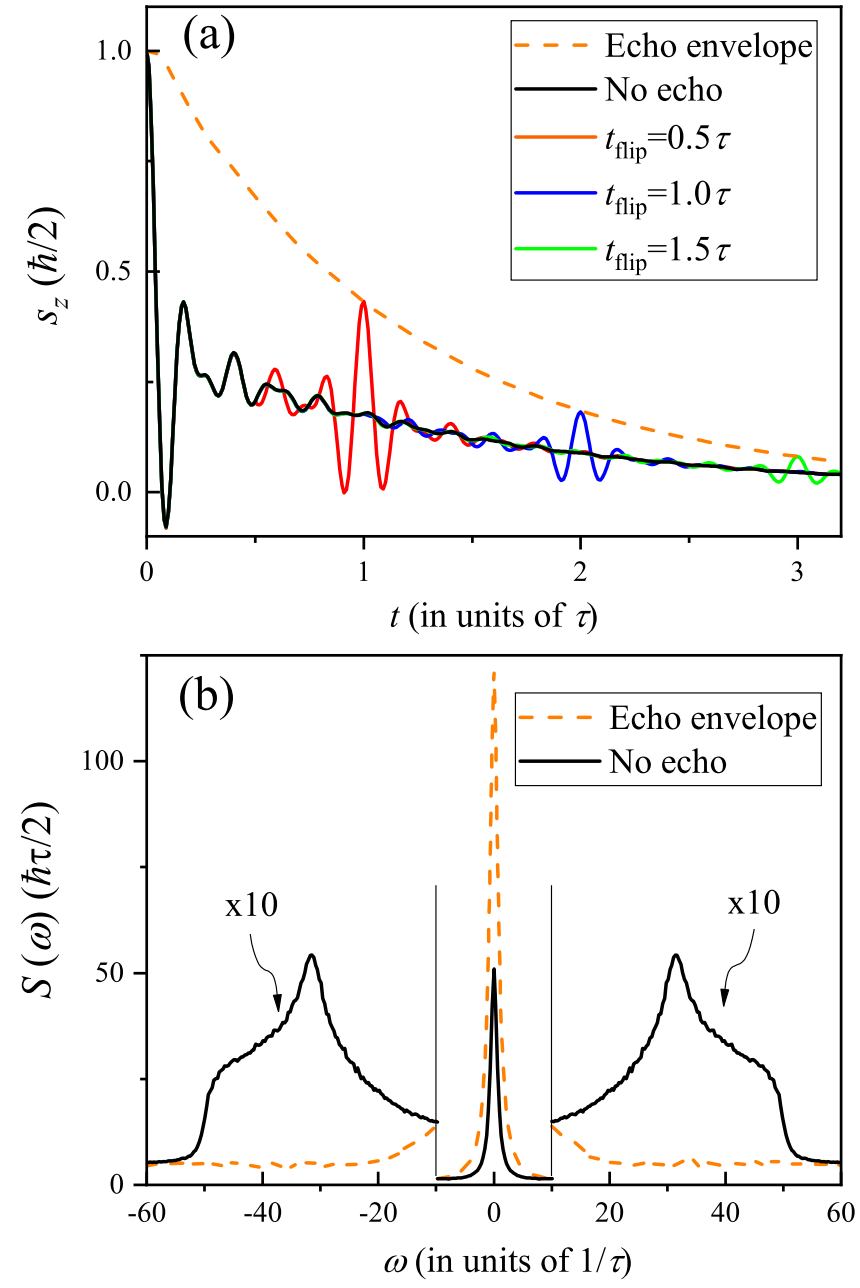

FIG. 4. (a) Spin decay for a spin ensemble which is spin polarized at $t=0$ (black solid line) and is under the action of a strong Dresselhaus SOC, which leads to reversible dephasing. Loschmidt echoes (red, blue, and green lines) are generated by inverting the Larmor frequency vectors at $t=\tau / 2, \tau$, and $1.5 \tau$ and these are observed around $t=\tau, 2 \tau$, and $3 \tau$, respectively. The envelope of these echoes is depicted with a dashed orange line. (b) Fourier transform of the spin decay and the Loschmidt echo envelopes. Note that the latter contains a single peak, centered at $\omega=0$, whereas the earlier has a peak at $\omega=0$ and two side-lobe structures (shown on a magnified scale), which reflect the Larmor frequency distribution in the Dresselhaus SOC.

distribution of the Larmor frequencies in the Dresselhaus SOC.

The simultaneous presence of reversible dephasing and relaxation is encountered in magnetic resonance and is tackled with the concept of spin echo [36,37], which is discussed in detail below. The spin echo is a specific case of the Loschmidt echo [26]. In our case, the Loschmidt echo can be numerically introduced by inverting the $\boldsymbol{\Omega}(\mathbf{k})$ vectors at a given instant and observing the recovery of the ensemble spin value. Figure 4(a) shows three such echoes which were generated by inverting the $\boldsymbol{\Omega}(\mathbf{k})$ vectors at three different instances. The envelope of the echoes is also shown in the figure. We developed a numerical method [38] to obtain the Loschmidt echo envelopes without needing to calculate the echoes at each time 
points. The corresponding Fourier transform, $\mathcal{S}(\omega)$, of the Loschmidt echo envelope is shown in Fig. 4(b): as expected, it shows a single peak at $\omega=0$ which contains all the spectral weight from the two side lobes. We highlight an interesting analogy of the present calculations with magnetic resonance: the timescale of reversible spin dephasing corresponds to $T_{2}^{*}$ (often referred to as reversible "relaxation time") and the envelope to the $T_{2}$ spin-spin relaxation time (also known as irreversible relaxation time).

Inverting the SOC-related Larmor frequency vectors is not practically conceivable in bulk solid state realizations. However, inverting an external electric field induced SOC, such as the Bychkov-Rashba SOC in two-dimensional heterolayers, may be feasible. Determination of the Loschmidt echo envelope allows us to determine the "true," i.e., irreversible spin-relaxation time. The data shows an exponential time dependence of the Loschmidt echo envelope over several orders of magnitude (shown in Ref. [38]) except for the beginning of the envelope for $\tau \ll 1$ where it starts with zero derivative due to geometric reasons.

\section{THE LOSCHMIDT ECHO IN MAGNETIC RESONANCE}

It is a common challenge in magnetic resonance that reversible dephasing and spin-relaxation processes are simultaneously present. The so-called spin echo, which is a realization of the Loschmidt echo, is employed to tackle this problem. Most generally, one encounters three different timescales in magnetic resonance: $T_{2}^{*}, T_{2}$, and $T_{1}$. Of these, $T_{1,2}$ are irreversible relaxation processes and $T_{2}^{*}$ is related to the reversible dephasing processes [36,37]. The distinction between $T_{1}$ and $T_{2}$ stems from the fact that a magnetic field is applied, which inevitably leads to a distinction between relaxation processes for the magnetization components which are parallel (the $T_{1}$ processes, also known as longitudinal relaxation time) and perpendicular (the $T_{2}$ processes, also known as transversal relaxation time) to the external magnetic field. In zero magnetic field, this distinction vanishes.

The Bloch equations [36] describe the motion of spins in a DC magnetic field, applied along the $z$ axis, which is accompanied by a circularly polarized AC magnetic field (with polarization plane perpendicular to $z$ ). In equilibrium, the magnetization of the spin ensemble, $\mathbf{M}$, is stationary along the $z$ axis with a value of $\mathbf{M}_{\mathbf{0}}$. When the AC magnetic field is applied in a pulsed manner, the magnetization is rotated away from the $z$ axis and starts to precess around $z$ with the Larmor frequency $\omega_{\mathrm{L}}=\gamma B$ (B is the magnetic field and $\gamma$ is the socalled gyromagnetic ratio of the studied spin system, e.g., $\gamma \approx$ $2 \pi \times 42.6 \mathrm{MHz} / \mathrm{T}$ for protons and $\gamma \approx 2 \pi \times 28.0 \mathrm{GHz} / \mathrm{T}$ for electrons).

In a typical experiment, an AC irradiation is applied with an angular frequency matching $\omega_{\mathrm{L}}$ and a pulse duration which is sufficient to rotate $\mathbf{M}$ into the $(x, y)$ plane. This is known as a $\pi / 2$ pulse, as the magnetization is rotated perpendicular to $z$. Then the $(x, y)$ and $z$ components of the nonequilibrium spin magnetization decay to the respective equilibrium values $\left(0\right.$ and $M_{0}$ ) with $T_{2}$ and $T_{1}$ relaxation times. However, in most cases the $(x, y)$ components vanish much earlier than $T_{2}$ due to reversible dephasing: local magnetic field inhomogeneities are present which lead to a distribution in $\omega_{\mathrm{L}}$. The inhomo-

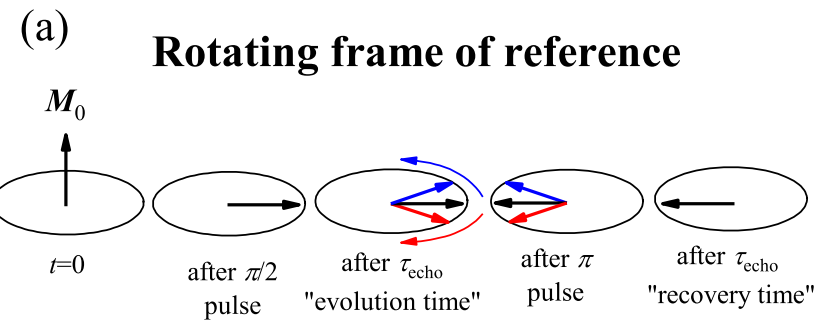

(b)

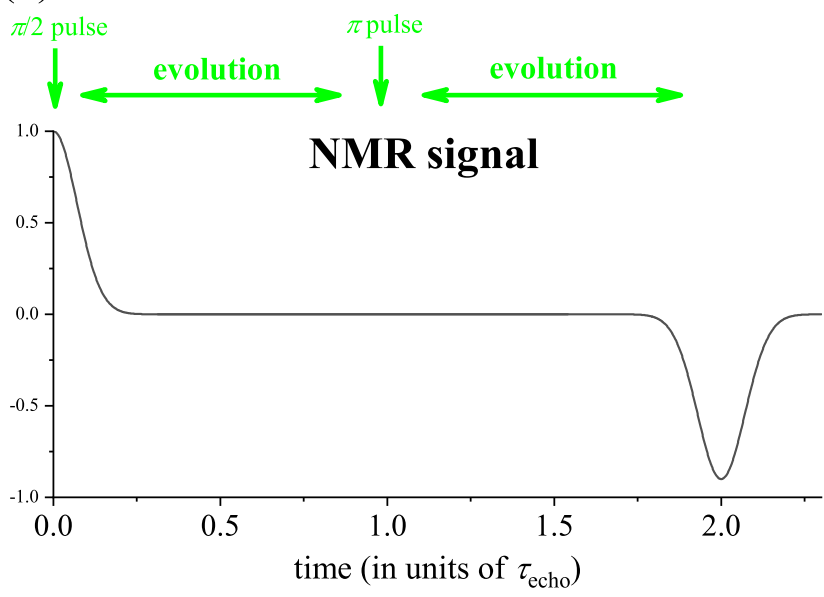

FIG. 5. (a) Schematics of the reversible dephasing process in NMR experiments and the method of spin echo. The figure assumes a right-handed precession direction with $\overline{\omega_{\mathrm{L}}}$. The spin magnetization lies in the plane perpendicular to $z$ after a $\pi / 2$ pulse when reversible dephasing due to a spread in the Larmor frequencies starts: spins in blue and red precess faster or slower than $\overline{\omega_{\mathrm{L}}}$, respectively. After an evolution time of $\tau_{\text {echo }}$, a $\pi$ pulse is applied which rotates the spins around an axis perpendicular to $z^{\prime}$. Clearly, the blue and red spins are now behind or before the average spin direction and as a result these are aligned coherently after another $\tau_{\text {echo }}$ time, when the spin echo occurs. (b) The corresponding NMR signal. Note the sign reversal of the NMR signal for this particular type of the spin echo.

geneities are caused by either defects or impurities (these are the leading cause in solid state NMR) or by the inevitable inhomogeneity of the magnet (this is the leading cause in high-resolution or liquid NMR) [37].

This process is usually described in a frame of reference which rotates with the mean value of the Larmor (angular frequency), $\overline{\omega_{\mathrm{L}}}$, around the $z$ axis and is schematically shown in Fig. 5(a). The coordinate axes of the rotating frame of reference are denoted by $x^{\prime}, y^{\prime}$, and $z^{\prime}\left(z^{\prime}\right.$ is identical to the $z$ axis). The $M_{0}$ magnetization lies in the $\left(x^{\prime}, y^{\prime}\right)$ plane after a $\pi / 2$ pulse, where reversible dephasing starts. In the rotating frame of reference, some spins have angular frequencies which are larger (the blue arrows in the figure) or smaller (the red arrows in the figure) than $\overline{\omega_{\mathrm{L}}}$. The resulting net magnetization vanishes on a timescale of $T_{2}^{*} \approx 1 / \Delta \omega_{L}$, where the latter is the spread in the Larmor frequencies. Another pulse is applied after a so-called "evolution time," $\tau_{\text {echo }}$, which rotates the spins by $\pi$. The figure depicts the location of the spins which precess faster (blue arrows) and slower (red arrows) than the average after the $\pi$ pulse. Clearly, after a waiting time of another $\tau_{\text {echo }}$ the spins are aligned again 

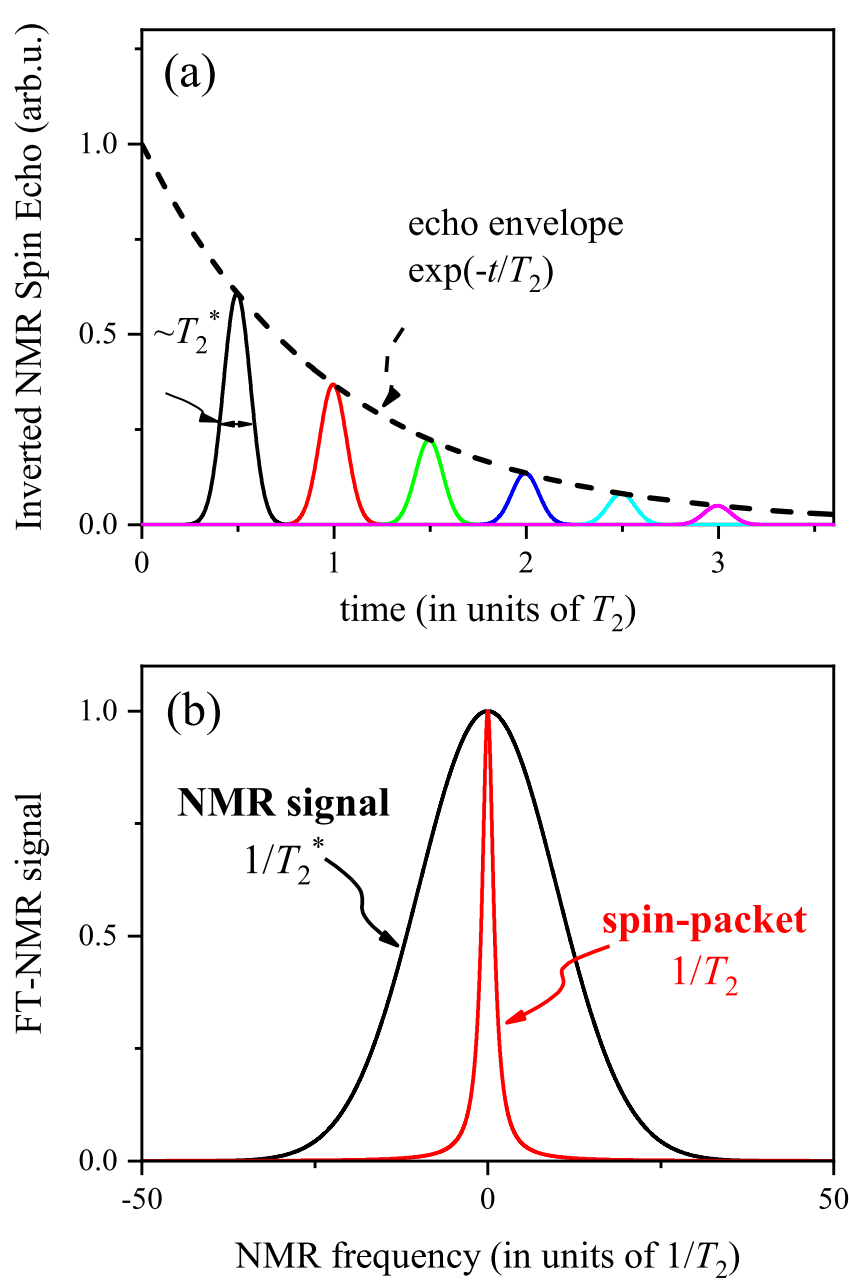

FIG. 6. (a) Schematics of the $T_{2}$ measurement. NMR spin-echo experiments are performed with varying time delay between the $\pi / 2$ and $\pi$ pulses. The individual echoes have a linewidth of $2 T_{2}^{*}$ but the resulting spin-echo envelope follows $e^{-t / T_{2}}$. (b) The corresponding Fourier transform (FT) NMR signal reflects this behavior: it contains a broad signal whose width is $1 / T_{2}^{*}$ and it consists of individual spin packets whose width is $1 / T_{2}$. Note the conceptual similarity of the present figure to Fig. 4.

coherently with a direction opposite to their original coherent direction.

Figure 5(b) also shows the corresponding NMR signal: the initially decaying signal is partially recovered, i.e., an echo is observed at $2 \tau_{\text {echo }}$ when the $\pi$ pulse is applied at $\tau_{\text {echo }}$. The reason why the NMR spin echo is observed, is that the reversible dephasing is not accompanied by a memory loss, thus each spin "remembers" the magnitude of its Larmor frequency. However in reality, memory loss is also present on the spin-relaxation timescale, $T_{2}$, where typically $T_{2}^{*} \ll$ $T_{2}$. In NMR, the physical origin of $T_{2}$ can be dipole-dipole interaction (this is the leading mechanism in solid state NMR) or molecular diffusion (this is the leading mechanism in highresolution or liquid NMR) [37].

Figure 6(a) shows the schematics of the $T_{2}$ measurement: spin-echo experiments are performed consecutively (in different pulse sequence runs, each starting from the equilibrium $M_{0} \| z^{\prime}$ ) with varying $\tau_{\text {echo }}$. The envelope of the observed echoes follows $e^{-t / T_{2}}$, which allows for the determination of $T_{2}$, which is a true, irreversible relaxation time, clearly distinguishable from the reversible dephasing. After Fourier transformation [shown in Fig. 6(b)], the NMR signal has a large linewidth of $1 / T_{2}^{*}$ (in frequency units) which consists of so-called spin packets [39], whose linewidth is $1 / T_{2}$. Clearly, both Figs. 6(a) and 6(b) are similar to Figs. 4(a) and 4(b), respectively.

\section{EFFICIENT CALCULATION OF THE LOSCHMIDT ECHO ENVELOPE IN OUR NUMERICAL STUDIES}

We outlined above how individual Loschmidt echoes can be obtained by inverting the SOC-related Larmor precession vectors. In principle, the envelope could be obtained from such individual echoes by varying the time delay of the reversal. Clearly, this procedure requires one to calculate a full ensemble-averaged time evolution and repeating this calculation over and over for each time-delay point. However, it turns out that the envelope itself can be obtained more effectively when we are not interested in the individual Loschmidt echoes. It turns out that this calculation is not more time-consuming than calculating a single time decay of the spin ensemble.

The schematics of the method is depicted in Fig. 7. It is based on keeping track of the rotation operator (which is a $3 \times$ 3 matrix), $U\left(t_{1}, t_{2}\right)$, which describes the evolution of a single spin at $t_{1}$ to a time point of $t_{2}$. Although momentum scattering happens in random time intervals, $U\left(t_{1}, t_{2}\right)$ can be constructed for any $t_{1}$ and $t_{2}$, which also involves the randomizing nature of the momentum scattering, which changes the direction of the Larmor precession. However, it is practical to predefine an equidistant array of time steps for which the envelope is to be calculated.

In addition to keeping track of the rotation operator under the action of the Larmor precession with randomized $\boldsymbol{k}$ values, we keep track of the rotation operators which would act for the inverted Larmor precession vectors. This is denoted by $U^{\prime}\left(t_{1}, t_{2}\right)$. Figure 7(a) depicts the action of these two types of operators by arrows.

Next, we consider an individual Loschmidt echo where the SOC-related Larmor vectors are inverted at a flip time of $t_{\text {flip. }}$. Figure 7(b) depicts that the echo can be obtained for any arbitrary time $t$ from the action of $U\left(0, t_{\text {flip }}\right)$, followed by $U^{\prime}\left(t_{\text {flip }}, t\right)$, i.e., their product. Figure $7(\mathrm{c})$ describes the efficient method to obtain the Loschmidt echo envelope at an arbitrary $t_{\text {echo }}=2 t_{\text {flip }}$. It requires the subsequent action of $U\left(0, t_{\text {flip }}\right), U^{\prime-1}\left(0, t_{\text {flip }}\right)$, and $U^{\prime}\left(0, t_{\text {echo }}\right)$ since the identity

$$
U^{\prime}\left(t_{\text {flip }}, 2 t_{\text {flip }}\right)=U^{\prime-1}\left(0, t_{\text {flip }}\right) U^{\prime}\left(0,2 t_{\text {flip }}\right)
$$

holds. Clearly this method involves a larger memory use but it substantially accelerates the calculations. In the end, the Loschmidt echo envelope for each individual spin needs to be ensemble averaged to obtain the final result.

Figure 8 shows the time dependence of the Loschmidt echo envelope and the spin-decay signal for longer times on a semilogarithmic plot (vertical axis is logarithmic). Note that both signals decay exponentially for longer times. The apparent noise in the signals for longer times could in principle be reduced by increasing the ensemble. 


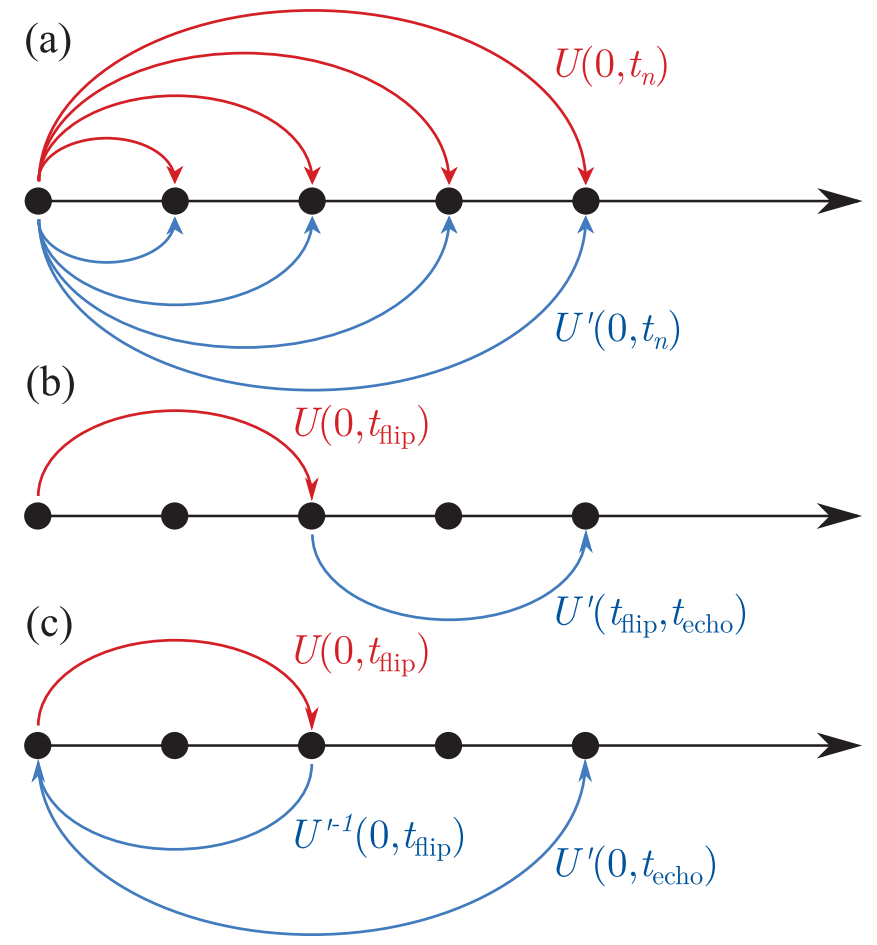

FIG. 7. Schematics of the efficient Loschmidt echo envelope calculation. (a) The Larmor precession acts as a transformation operator, $U\left(t_{1}, t_{2}\right)$, on an individual spin. This can be obtained for an equidistant array of time values, even in the presence of momentum scattering which provides a new random $\boldsymbol{k}$ that alters the spin precession direction. $U^{\prime}\left(t_{1}, t_{2}\right)$ is obtained for the inverted Larmor precession vectors. (b) The time trace of an individual echo with flip time $t_{\text {flip }}$ could be obtained at any time $t$ by acting on the spin with $U\left(0, t_{\text {flip }}\right)$, followed by $U^{\prime}\left(t_{\text {flip }}, t\right)$. (c) The echo envelope at a time point $t_{\text {echo }}=2 t_{\text {flip }}$ is obtained from the product $U\left(0, t_{\text {flip }}\right) U^{\prime-1}\left(0, t_{\text {flip }}\right) U^{\prime}\left(0, t_{\text {echo }}\right)$.

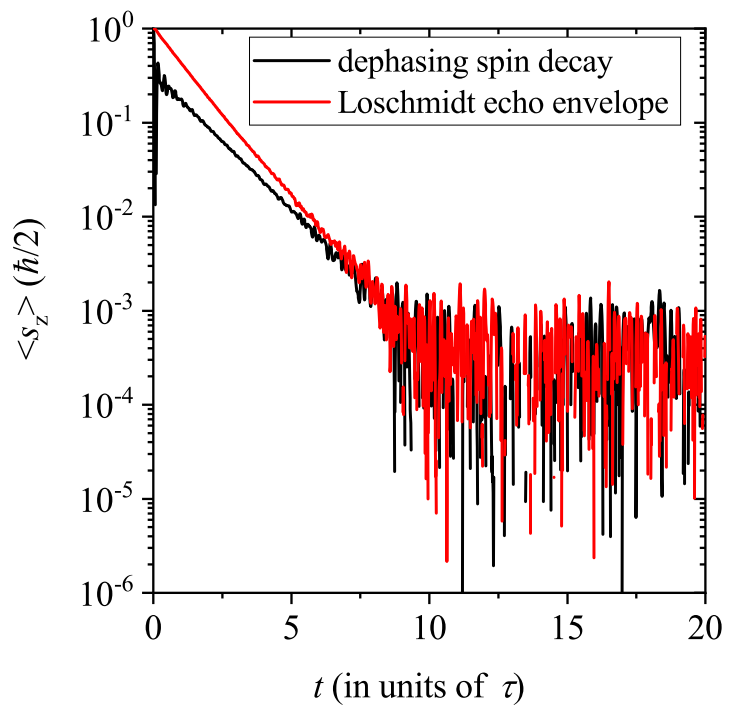

FIG. 8. Time dependence of the Loschmidt echo envelope and the spin-decay signal for longer times on a semilogarithmic plot. Note the exponential decay for both the spin-decay and the Loschmidt echo envelope signals.
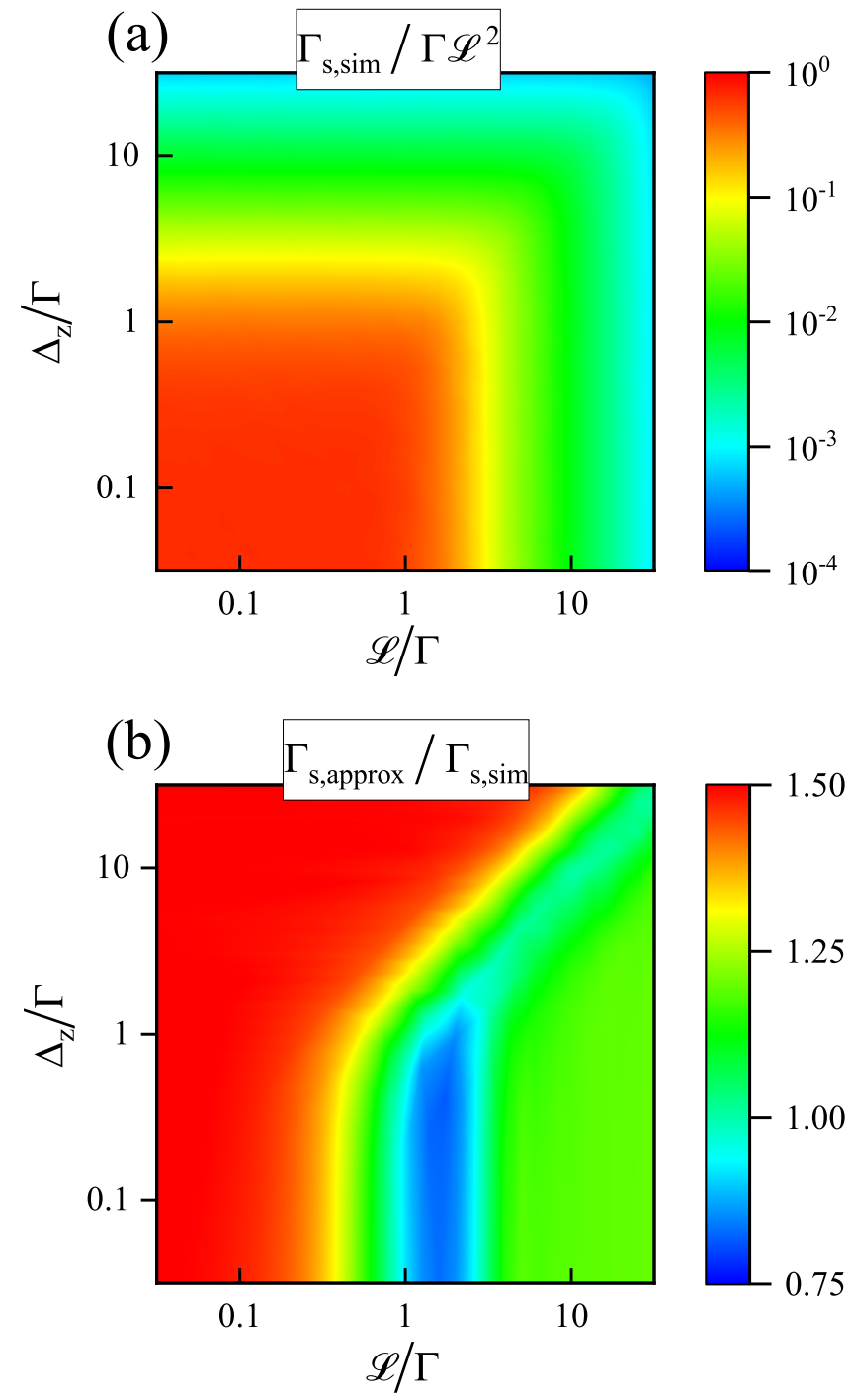

FIG. 9. (a) Phase diagram of the spin-relaxation rate, $\Gamma_{\mathrm{s}, \mathrm{sim}}$, for the Dresselhaus SOC as a function of $L / \Gamma$ and $\Delta_{\mathrm{Z}} / \Gamma$. The data are normalized by $\Gamma L^{2}$. (b) Ratio of the approximate generic formula of $\Gamma_{\mathrm{s}, \text { approx }}=\frac{\Gamma L^{2}}{\Gamma^{2}+L^{2}+\Delta_{\mathrm{Z}}^{2}}$ and $\Gamma_{\mathrm{s}, \mathrm{sim}}$. Note that this ratio is around unity, which justifies the use of the generic formula.

\section{A. The generic phase diagram of spin relaxation}

The calculated spin-relaxation rate, $\Gamma_{\mathrm{s}, \mathrm{sim}}$, is plotted for the entire phase diagram as a function of $L / \Gamma$ and $\Delta_{\mathrm{Z}} / \Gamma$ in Fig. 9(a). $\Gamma_{\mathrm{s} \text {,sim }}$ values are obtained by fitting exponential time dependences to the Loschmidt echo envelopes, which are obtained from our MC calculations. The data are normalized by $\Gamma L^{2}$; without this normalization, the value of $\Gamma_{\mathrm{s} \text {,sim }}$ changes by eight orders of magnitude for the given range. We note that the cyclotron orbital motion of $\mathbf{k}$ in high magnetic field was neglected in the calculations; however, we believe that this could be straightforwardly implemented.

Figure 9(b) shows the ratio of the suggested generic formula $\Gamma_{\mathrm{s} \text {,approx }}=\frac{\Gamma L^{2}}{\Gamma^{2}+L^{2}+\Delta_{Z}^{2}}$ and $\Gamma_{\mathrm{s} \text {,sim }}$ obtained from the MC calculations. A good agreement is found between the $\mathrm{MC}$ simulations and the generic formula for the entire phase diagram, the difference not being larger than a factor of 2 . This 


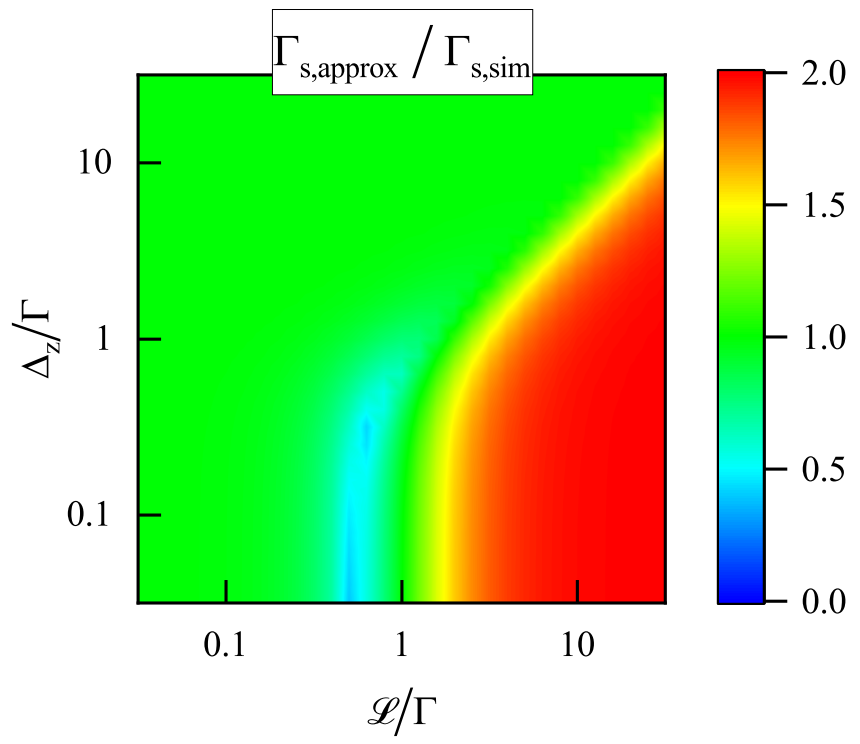

FIG. 10. Ratio of the recommended generic formula for the spinrelaxation rate and that obtained analytically for the $2 \mathrm{D}$ electron gas with the Bychkov-Rashba SOC.

strongly argues for the existence of a generic formula and for the validity of the presently suggested form. We believe that a similar formula is valid for an arbitrary SOC distribution while some multiplying factors (around unity) can be present in it.

\section{B. Validation for an analytically solvable model}

We also verified the validity of the suggested generic formula for a specific spin-relation example which is exactly solvable. Burkov and Balents [40] studied a two-dimensional electron gas with a lateral electric field, which induces a Bychkov-Rashba-type SOC. They presented an analytic result for the spin-relaxation rate using a many-body approach for arbitrary values of $\Gamma, L$, and $\Delta_{Z}$. Details of the analytic calculations in the various regimes are somewhat involved and are presented in the Supplemental Material [38]. In Fig. 10, we show the ratio between the approximation formula in the main text and the herein presented simulated values. The agreement between the two kinds of data is close to unity and does not deviate from it more than a factor 2 .

This agreement provides additional support for the validity of the recommended generic formula. We believe that besides the aforementioned multiplying factors, the formula may serve with a strong predicting value for the spinrelaxation and spin-transport $[41,42]$ properties in future spintronics materials. In addition, it describes well the general trends for the spin-relaxation rate versus its parameters, which can help to identify the relevant model of a relaxation mechanism. Although we did not cover the case of spin relaxation in systems with inversional symmetry (the Elliott-Yafet theory $[8,9])$, the recently discovered equivalence between the Dyakonov-Perel and Elliott-Yafet Hamiltonians [12] allows for a straightforward application of the present result for the Elliott-Yafet case.

\section{CONCLUSIONS}

In conclusion, we studied the spin dynamics in zincblende semiconductors with the Dresselhaus spin-orbit coupling. We presented a model, which directly provides the quantum trajectories of individual spins and is equivalent to solving the spin Boltzmann equation. The method could be readily generalized for interacting electrons where its advantage, in terms of calculation efficiency, prevails. We identified a nonexponential, spin-dephasing regime of spin dynamics, which occurs due to a strong SOC. We tackled reversible dephasing with the introduction of a Loschmidt echo. This allowed us to determine the spin-relaxation time for the entire spin-relaxation phase diagram involving the strength of the quasiparticle scattering rate, spin-orbit coupling, and the Zeeman interaction. We found that a simple and compact form approximates well $\tau_{\mathrm{s}}$. The validity of the formula was also confirmed for the two-dimensional electron gas with a lateral electric field (i.e., with a Bychkov-Rashba SOC) for which the spin-relaxation time could be determined analytically.

\section{ACKNOWLEDGMENTS}

The authors are indebted to Jaroslav Fabian for many stimulating discussions. This work is supported by the Hungarian National Research, Development and Innovation Office (NKFIH) Grants No. 2017-1.2.1-NKP-2017-00001, No. K124176, and No. K119442. A.K. acknowledges the Bolyai Program of the Hungarian Academy of Sciences. F.S. acknowledges the hospitality of L. Forró during a sabbatical stay and financial support by the Swiss National Science Foundation (Grants No. 200021 and No. 144419) and European Research Council (ERC) Advanced Grant No. 670918.
[1] S. A. Wolf, D. D. Awschalom, R. A. Buhrman, J. M. Daughton, S. von Molnár, M. L. Roukes, A. Y. Chtchelkanova, and D. M. Treger, Science 294, 1488 (2001).

[2] I. Žutić, J. Fabian, and S. Das Sarma, Rev. Mod. Phys. 76, 323 (2004).

[3] M. W. Wu, J. H. Jiang, and M. Q. Weng, Phys. Rep. 493, 61 (2010).

[4] K. S. Novoselov, A. K. Geim, S. V. Morozov, D. Jiang, Y. Zhang, S. V. Dubonos, I. V. Grigorieva, and A. A. Firsov, Science 306, 666 (2004).
[5] N. Tombros, C. Józsa, M. Popinciuc, H. T. Jonkman, and B. J. van Wees, Nature (London) 448, 571 (2007).

[6] W. Han, K. Pi, K. M. McCreary, Y. Li, J. J. I. Wong, A. G. Swartz, and R. K. Kawakami, Phys. Rev. Lett. 105, 167202 (2010).

[7] T.-Y. Yang, J. Balakrishnan, F. Volmer, A. Avsar, M. Jaiswal, J. Samm, S. R. Ali, A. Pachoud, M. Zeng, M. Popinciuc et al., Phys. Rev. Lett. 107, 047206 (2011).

[8] R. J. Elliott, Phys. Rev. 96, 266 (1954).

[9] Y. Yafet, Solid State Phys. 14, 1 (1963). 
[10] M. I. Dyakonov and V. I. Perel, Fiz. Tverd. Tela 13, 3581 (1971) [Sov. Phys. Solid State 13, 3023 (1971)].

[11] G. E. Pikus and A. N. Titkov, Spin Relaxation Under Optical Orientation in Semiconductors (Elsevier, Amsterdam, 1984), pp. 73-131.

[12] L. Szolnoki, B. Dóra, A. Kiss, J. Fabian, and F. Simon, Phys. Rev. B 96, 245123 (2017).

[13] M. Krauß, M. Aeschlimann, and H. C. Schneider, Phys. Rev. Lett. 100, 256601 (2008).

[14] P. Zhang and M. Wu, New J. Phys. 14, 033015 (2012).

[15] O. D. Restrepo and W. Windl, Phys. Rev. Lett. 109, 166604 (2012).

[16] T. Yu and M. W. Wu, Phys. Rev. B 93, 045414 (2016).

[17] L. Wang and M. W. Wu, Phys. Rev. B 89, 115302 (2014).

[18] P. Boross, B. Dóra, A. Kiss, and F. Simon, Sci. Rep. 3, 3233 (2013).

[19] L. Szolnoki, A. Kiss, B. Dóra, and F. Simon, Sci. Rep. 7, 9949 (2017).

[20] J. M. Kikkawa and D. D. Awschalom, Phys. Rev. Lett. 80, 4313 (1998).

[21] D. D. Awschalom, Phys. E (Amsterdam, Neth.) 10, 1 (2001).

[22] M. Wu and C. Ning, Physica Status Solidi B 222, 523 (2000).

[23] M. W. Wu, J. Supercond. 14, 245 (2001).

[24] M. A. Brand, A. Malinowski, O. Z. Karimov, P. A. Marsden, R. T. Harley, A. J. Shields, D. Sanvitto, D. A. Ritchie, and M. Y. Simmons, Phys. Rev. Lett. 89, 236601 (2002).

[25] W. J. H. Leyland, R. T. Harley, M. Henini, A. J. Shields, I. Farrer, and D. A. Ritchie, Phys. Rev. B 76, 195305 (2007).

[26] A. Goussev, R. A. Jalabert, H. M. Pastawski, and D. A. Wisniacki, Scholarpedia 7, 11687 (2012), revision \#127578.

[27] E. L. Hahn, Phys. Rev. 80, 580 (1950).
[28] K. W. Murch, S. J. Weber, C. Macklin, and I. Siddiqi, Nature (London) 502, 211 (2013).

[29] Q. Ficheux, S. Jezouin, Z. Leghtas, and B. Huard, Nat. Commun. 9, 1926 (2018).

[30] Z. K. Minev, S. O. Mundhada, S. Shankar, P. Reinhold, R. Gutiérrez-Jáuregui, R. J. Schoelkopf, M. Mirrahimi, H. J. Carmichael, and M. H. Devoret, Nature (London) 570, 200 (2019).

[31] J. Fabian, A. Matos-Abiaguea, C. Ertlera, P. Stano, and I. Zutic, Acta Phys. Slov. 57, 565 (2007).

[32] K. Mølmer, Y. Castin, and J. Dalibard, J. Opt. Soc. Am. B 10, 524 (1993)

[33] H.-P. Breuer and F. Petruccione, The Theory of Open Quantum Systems (Oxford University, New York, 2006).

[34] V. K. Dugaev, E. Y. Sherman, and J. Barnaś, Phys. Rev. B 83, 085306 (2011).

[35] S. Kudła, A. Dyrdał, V. K. Dugaev, E. Y. Sherman, and J. Barnaś, Phys. Rev. B 97, 245307 (2018).

[36] C. P. Slichter, Principles of Magnetic Resonance, 3rd ed. (Spinger-Verlag, New York, 1989)

[37] A. Abragam, Principles of Nuclear Magnetism (Oxford University Press, Oxford, England, 1961).

[38] See Supplemental Material at http://link.aps.org/supplemental/ 10.1103/PhysRevResearch.2.033058 for additional discussion on the spin Boltzmann equation and the Loschmidt echo.

[39] A. M. Portis, Phys. Rev. 91, 1071 (1953).

[40] A. A. Burkov and L. Balents, Phys. Rev. B 69, 245312 (2004).

[41] S. V. Iordanskii, Y. B. Lyanda-Geller, and G. E. Pikus, J. Exp. Theor. Phys. 60, 206 (1994).

[42] W. Knap, C. Skierbiszewski, A. Zduniak, E. LitwinStaszewska, D. Bertho, F. Kobbi, J. L. Robert, G. E. Pikus, F. G. Pikus, S. V. Iordanskii et al., Phys. Rev. B 53, 3912 (1996). 